\title{
Interferometric characterization of GRIN lenses for ophthalmic uses
}

\author{
J. R. de F. Moneo, I. Juvells, S. Vallmitjana, S. Bosch, A. Carnicer, I. Labastida and J. Pérez \\ Universitat de Barcelona, Laboratori d'Optica, Departament de Física Aplicada i Electrònica \\ Diagonal 647, E08028 Barcelona (Spain) E-mail: sbp@hermes.fae.ub.es
}

\begin{abstract}
The proposal of this work is the study of a method for characterization of the focal of ophthalmic lenses whose surface refraction index has been modified by diffusion techniques based on thermal ion exchange in glass. In particular the aim is the measurement of the variations in focal length of ophtalmic lenses after undergoing a diffusion process. An interferometric technique has been used and computer simulations have also been carried out.
\end{abstract}

\section{Introduction}

The inclusion of gradient-index technology in optical elements has become a very impresive field in applied optics. The possibility of totally or partially cahnging the index of refraction in lenses designed for vision purposes is a very attrative subject. The proposal of this work is the study of a method for characterization ophthalmic lenses of which the surface refraction index has been modified by diffusion techniques based on thermal ion exchange in glass. In particular the aim is the measurement of the variations in focal length of ophtalmic lenses after undergoing a diffusion process. As these variations are extremely small, an interferometric technique is needed. Among the different architectures of lateral shearing interferometers we have chosen the Mach-Zehnder interferometer (MZI) as a powerful tool for optical testing. ${ }^{1,2}$ A method for the calculation of the focal length derived from the interferograms is described. In order to obtain the best work conditions and to test the method of measurement, a computer simulation has been also carried out.

\section{Method}

The method of calculation of the focal length is based on the analysis of the interference pattern between a plane wave and the corresponding spherical wave after passing through the lens. This interference is carried out by means of a MZI and is recorded through a CCD camera and a frame grabber.

Once the focal length of the test lens has been calculated, the surface index of this lens is modified by a diffusion technique and a new measurement of the focal length is realized by the analysis of the interference pattern. The final difference between both calculated focal lengths will give the influence of the gradient index variation on the lens.

The basis of the calculation of the focal length (see Figure 1) is the obtention of the distance $z$, which is the distance between the interference plane and the center of the conververging spherical wave. The distance between the back principal plane of the lens and the interference plane is $d$. The addition of $\mathrm{z}$ plus $\mathrm{d}$ will give this focal, as can be seen in the same Figure. In the setup, a plane wave interferes with the converging one to its focus, giving concentric circles, as seen in Figure 2. 


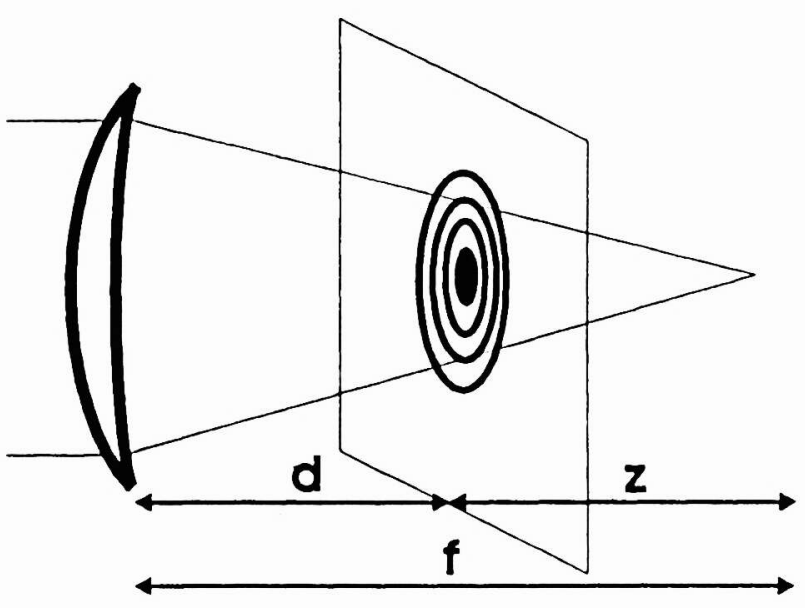

Figure 1: Focal length measurement

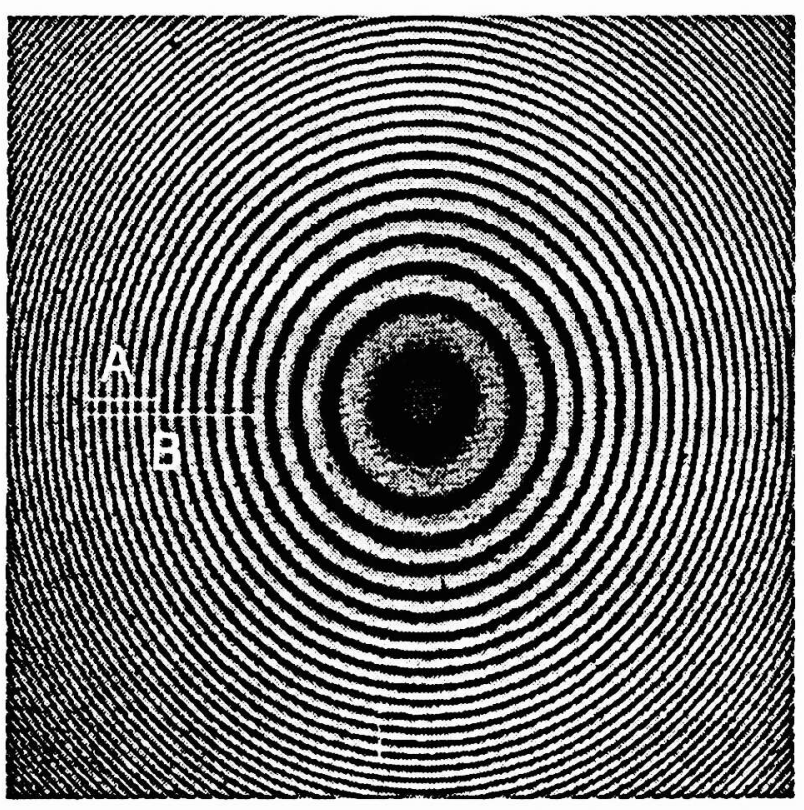

Figure 2: $\mathrm{A}$ and $\mathrm{B}$ measurement

By analyzing the interference rings between a plane wave and a spherical wave converging (or diverging) to the distance $z$ behind (or before) the plane, the radius of the interference circles follow the relation ${ }^{3}$ :

$$
\rho_{n}^{2}=2 n \lambda z
$$

where $n$ is the interferometric order of the ring. Theoretically, measuring the radius of the rings in the interference plane would be enough to obtain $z$. This is not correct because the last equation is only true when the phase at the centre is zero (or there is a maximum at the centre). In general, the correct equation which gives the relation between radius of maxima of order $n$ and $z$ is:

$$
\rho_{n}=2 \lambda z(n \pm p) \quad \text { where } \quad 0 \leq p<1
$$

This now gives an extra unknown $p$, but moreover there is another difficulty that appears when $z$ is large. This case implies a large value of the radius which in the practical case could be longer than the actual size of the captured area taken by the CCD and the frame grabber. Another practical problem arises for the determination of the centre of the radius on the pixelated images because the circles are in grey levels and they are not perfect circumferences. As a result, all these experimental problems lead to make a very inaccurate measurement of $z$.

In order to overcome these drawbacks we propose a method of measuring based on substraction so, the central phase does not affect the results and, in addition, for large $z$ the CCD can be laterally displaced. This gives more rings regardless of the situation of the centre.

Starting from a certain ring (Figure 2), which is of a unknown order $n$, and measuring the distances $A$ and $B$, between $m$ and $2 m$ rings, we obtain,

$$
\begin{gathered}
\rho_{n}-\rho_{n-m}=A=\sqrt{2 \lambda z n}-\sqrt{2 \lambda z(n-m)} \\
\rho_{n}-\rho_{n-2 m}=B=\sqrt{2 \lambda z n}-\sqrt{2 \lambda z(n-2 m)}
\end{gathered}
$$


Solving the system:

$$
\begin{array}{r}
\frac{m}{n}=\frac{12 A B-8 A^{2}-4 B^{2}}{4 A B-4 A^{3} / B-B^{3} / A} \\
z=\frac{A^{2}}{2 \lambda n\left(1-\sqrt{1-\frac{m}{n}}\right)^{2}}
\end{array}
$$

To this distance $z$ (from the interference plane to focus) the distance from the lens to the interference plane has to be added, in order to obtain the focal length. For simplicity in the latter equations, we have supposed that there is a maximum in the centre (initial phase $p$ zero). In the general case (see equation 2) the integer corresponding to the order must be substituted by a real number $n \pm p$, but the system 3 remains the same and now $\mathrm{n}$ is real. It is important to remark that once the system is solved, Equation 2 is useful for checking and refining the results.

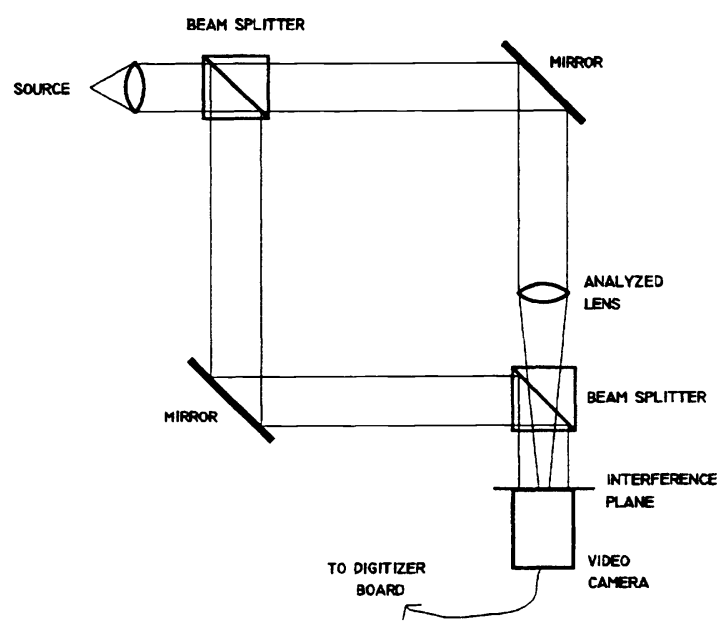

Figure 3: Mach-Zehnder interferometer

\section{Experimental}

\subsection{Setup}

This interference is carried out by means of a MZI on an isolated optical table, the arrangement of which is represented in Figure 3. By adjusing the collimator a coherent plane wave coming from a He-Ne laser crosses the first cube beam-splitter. In one of the arms the lens under study is placed and the wave plane is changed into convergent or divergent after passing through. In the other arm the plane wave remain the same. The interference between the two arms in the exit of the cube beam-splitter is grabbed in a 8 bit by means of a CCD camera.

\subsection{Optimization of the precission}

In this method it is very important to minimize all sources of error. First of all the optical quality must be good enough to avoid unwanted deformations of the wavefronts. In particular the resolution has to be high in the 
digitized rings. Theoretically, the longer the distances $A$ and $B$ measured the better precision in the results, but also the imprecision on the fainter and more concentrated rings affects negatively. Conversely if shorter distances are measured the results also begins to decay. In order to deal with this, an optimum range of distances has been found after a study based on a series of measurements over known tests. The exact situation of the maxima is another important element to be defined which also depends on the resolution of the frame grabber.

In order to reduce to the minimum the influence of these sources, a good $\lambda / 20$ was used in the mirrors, $\lambda / 10$ in the cubes, a special collimator and a microscope objective corrected for infinity was used on a compressed air active-isolated optical table. After trial error tests we have found an optimal compromise between the digitation, the precision and the counted number of rings. Other error sources due to the experimental limitations are derived from the fact that each lens is measured twice (before and after the difusion) and the conditions of the interferometer are not the same. These sources are: interference pattern without lens, conditions of room temperature, conditions of illumination and saturation of the CCD. Other problems arise from the fact that the digitation is squared over a rectangular sensitive surface.

Finally, in order to deal with these other sources, the same experimental protocol was used each time, and checking first the room conditions and the initial interference pattern, its verticality, and the photometric conditions.

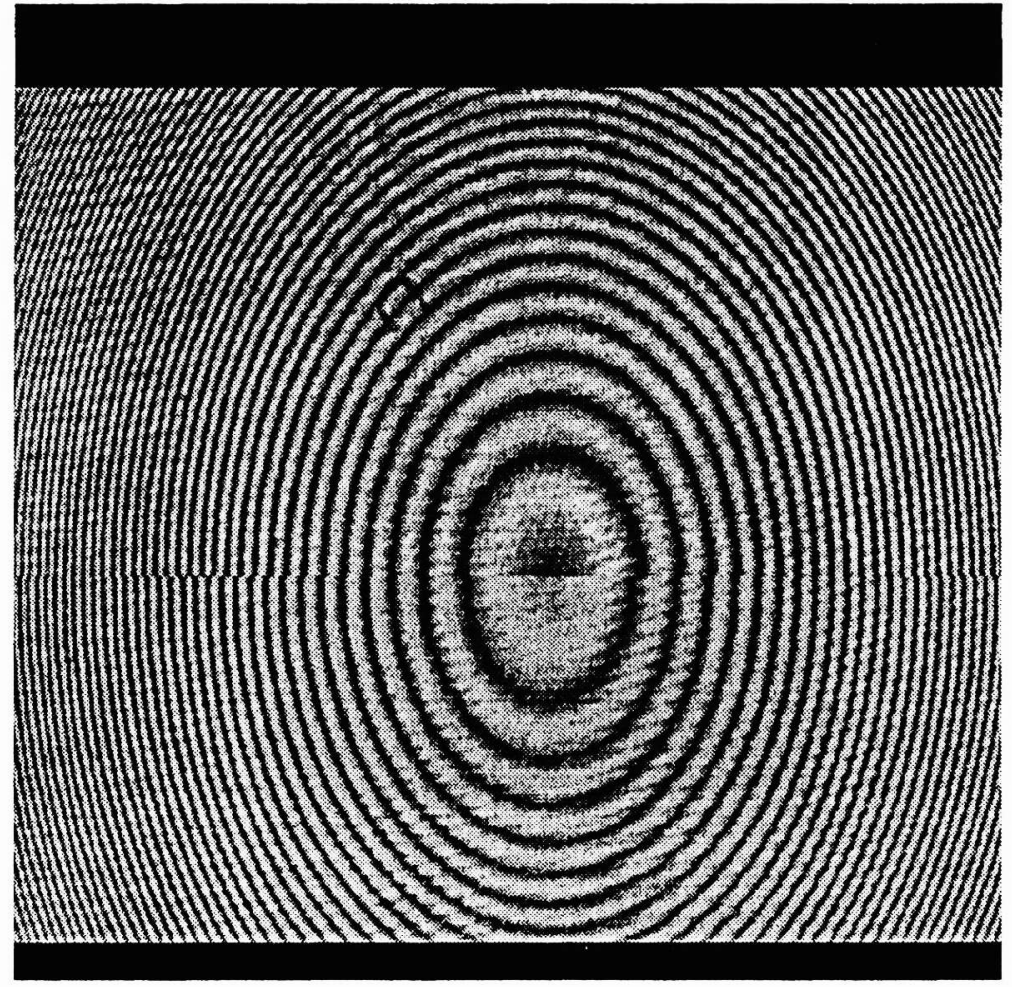

Figure 4: Superposition of two interferograms

\section{Results}

For each ophtalmic lens the procedure has been the same. First the lens is placed in the MZI within the aforemetioned coditions and from its interferogram the focal length is measured. Afterwards the lens is treated 
by diffusion techniques based on thermal ion exchange with a view to changing the surface refraction index. A second measurement in the MZI gives the variation on the focal length. Figure 4 shows the superposition of two interferograms of the same lens before (upper part) and after (lower) the diffusion bath. The fringe displacement is due more to the different central initial phase than to the difference on the gradient of the refraction index. In all the experiments the variations on the surface index of refraction give unaffected values of the power of the lens. The appreciated increments are so faint that they are inside of the range of imprecission. Our initial precision was a dispersion of \pm 0.025 dioptres over several measurements of a positive meniscus lens of a power of +2 dioptres. Now we have improved the method of measurement giving a dispersion of \pm 0.010 dioptres around the power tested.

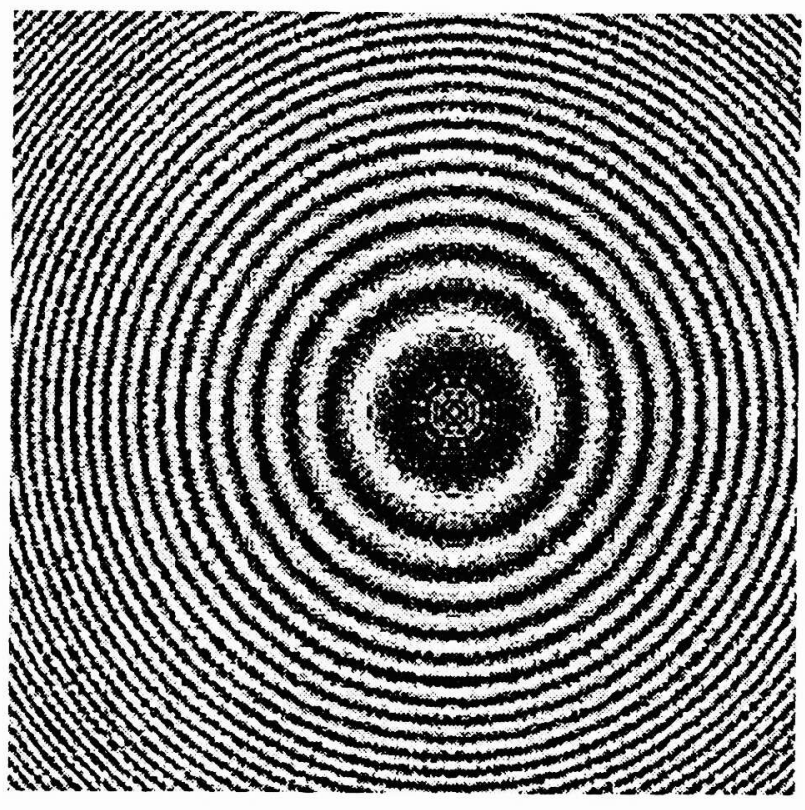

Figure 5: Simulated interferogram

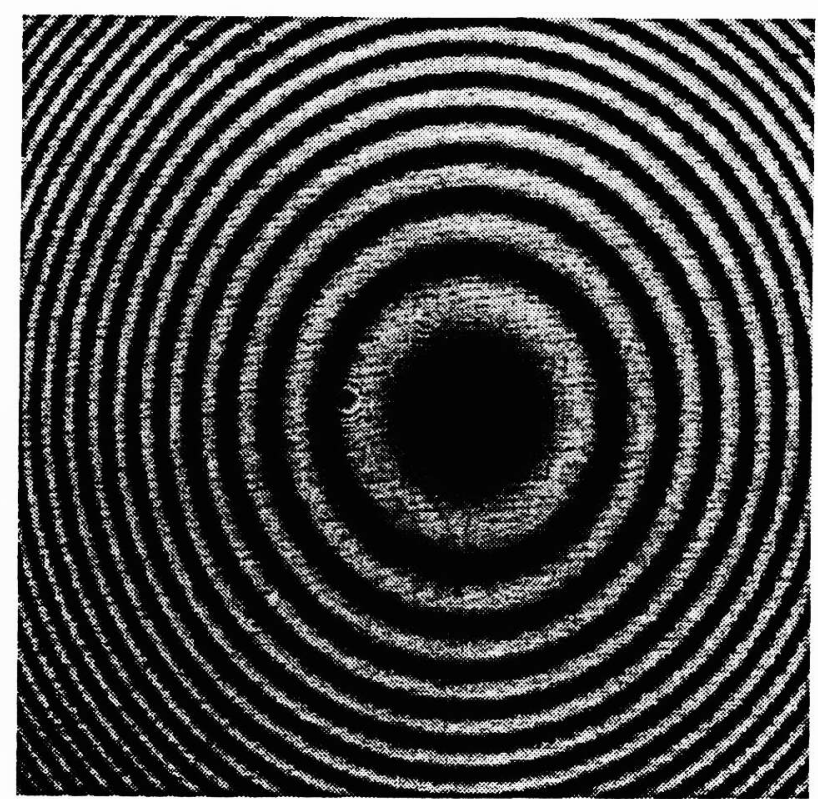

Figure 6: Experimental interferogram

\section{Simulated results}

Another way to test the method of obtaining the focal length has been carried out by simulation. A computer program which simulates the MZI has been designed. The parameters of the ophtalmic lens as curvature radii, index of refiaction and thickness are introduced. The program calculates and displays the simulated interferogram after entering the exact position of the lens. By measuring the same distances A and B over the rings as in the experiment, the program computes the focal power. In order to compare and simulate the experimental conditions, two meniscus of +1 and +2 dioptres (nominal value) are measured in the $\mathrm{MZ}$ interferometer. The two curvature radii and thickness were measured through a precision spherometer $( \pm=.001 \mathrm{~mm})$ and a precise value of the refraction index is given by the commercial supplier. With all these values the program in the computer calculates the interference rings and display the interferogram, with an accurated scale factor. The distances A and B are measured by the same method and with the same m number, finally a focal length results for the simulated interferometer. Figures 5 and 6 show respectively the simulated and the experimental interferograms obtained from the same lens. In the simulated interferogram the distances $A$ and $B$ are measured by the same method and with the same $m$ number. The results are as follows: 
1. Lens of +1 dioptre (nominal)

- Simulation: Obtained value: 0.958 dioptres

- Actual measurement in the MZI 0.967 dioptres.Error 0.009

2. Lens of +2 dioptre (nominal)

- Simulation: Obtained value: 1.960 dioptres

- Actual measurement in the MZI 1.979 dioptres. Error 0.019

The accordance is excellent because the difference between the measured and simulated values are within the tolerable range of precision according to the data values and the experimental method.

\section{Conclusions}

An interferometric method for obtaining the power focal of an ophthalmic lens with a precision better than one hundreth of dioptre has been developed. The method is based on the analysis of the interference pattern between a plane wave and the corresponding spherical wave after passing through the lens. This interference is obtained in a Mach Zehnder interferometer and recorded through a CCD and a 8 bit frame grabber. A computer programme that simulates the interferometric behaviour has also been developed. The method has been applied to measuring the variations of the focal power of a lens after undergoing a thermal ion exchange treatment in order to modify the surface variations of the refraction index.

\section{Acknowledgements}

This work has been supported by INDO, S.A., under project FBG/2216-10/95.

\section{REFERENCES}

[1] D. T. Moore and D. P. Ryan, "Measurement of the optical properties of gradient index materials", J. Opt. Soc. Am. 68, 1157-1166 (1978).

[2] H. El-Kashef, G. E. Hassan and I. El-Ghazaly, "Mach-Zehnder optical system as a sensitive measuring instrument", Appl. Opt. 33, 3540-3544 (1994).

[3] D. Malacara, "Optical Shop Testing", John Wiley Sons, New York (1978). 